\title{
universidâd
}

\section{Toxoplasmosis ocular reporte de caso y revisión de la}

\section{literatura}

\section{Ocular toxoplasmosis case report and literature review}

DOI: http://dx.doi.org/10.5377/uyc.v10i16.6136

Arlin Montoya,

montoyaarlin@gmail.com

Universidad Nacional Autónoma de Nicaragua, Managua

Mario Mayorga

Universidad Nacional Autónoma de Nicaragua, Managua

Clara González Moncada.

klarisabel@hotmail.com

Universidad Nacional Autónoma de Nicaragua, Managua

(C) UNAN-Managua

Recibido: 20 julio 2016. Aprobado: octubre 2017

\section{RESUMEN}

La toxoplasmosis ocular es una enfermedad causada por el parasito protozoario toxoplasma gondii. Aproximadamente un tercio de la población mundial está infectada por este patógeno, siendo generalmente una afectación asintomática. La afectación ocular se ha estimado que se produce en un $1-2 \%$ de las personas infectadas y se asocia con un riesgo de recurrencia de por vida y perdida de la visión, siendo poco frecuente esta presentación en paciente inmunocompetentes, por lo que se presenta el siguiente reporte de caso descriptivo. Paciente de 19 años asiste al CENAO con motivo de consulta; visión borrosa e hiperemia conjuntival, al fondo de ojo se observa zona activa de retinitis adyacente a la placa de coriorretinitis. En estudios de laboratorio se encontró Toxotest positivo con títulos $1 / 256$, Toxo IgM Negativo, Toxo IgG $322.6 \mathrm{UI} / \mathrm{ml}$, PCR Negativo, RPR No reactivo, VSG $4 \mathrm{~mm} / \mathrm{hr}$, HIV No reactivo, CMV IgM negativo, CMV IgG IgG 7.8 $\mathrm{UI} / \mathrm{ml}$. Lo particular de este caso es que es un paciente inmunocompetente, diagnosticado en etapa temprana y con resolución completa de su visión, lo que es una premisa para concluir que un diagnóstico temprano conlleva a un mejor pronóstico de la enfermedad.

Palabras Clave: Toxoplasmosis ocular, Toxoplasma gondii, Inmunocompetente

\section{SUMMARY}

Ocular toxoplasmosis is a disease caused by the protozoan parasite toxoplasma gondii. Approximately one-third of the world's population is infected by this pathogen, usually being asymptomatic. Ocular involvement has been estimated to occur in 1-2\% of infected persons and is associated with a lifetime risk of recurrence and loss of vision, and this presentation is uncommon in immunocompetent patients. the following descriptive case report. A 19-year-old patient attends CENAO for consultation; blurred vision and conjunctival hyperemia, the active eye zone of retinitis adjacent to the chorioretinitis plaque is observed in the fundus. In laboratory studies, Toxotest was positive with titles $1 / 256$, Toxo IgM Negative, Toxo IgG 322.6 IU / ml, PCR Negative, RPR Not reactive, VSG $4 \mathrm{~mm} / \mathrm{hr}$, HIV non-reactive, CMV IgM negative, CMV IgG IgG 7.8 UI / $\mathrm{ml}$. The particular case of this case is that it is an immunocompetent patient, diagnosed at an early stage and with complete resolution of his vision, which is a premise to conclude that an early diagnosis leads to a better prognosis of the disease. 
Keywords: Toxoplasmosis ocular, Toxoplasma gondii, Immunocompetent

\section{INTRODUCCIÓN}

La toxoplasmosis ocular es una enfermedad causada por el parasito protozoario Toxoplasma gondii. Las infecciones pueden ser adquiridas congénitamente o por medio de la ingestión de carne cruda, verduras contaminadas o agua (Dodds, 2003). Aproximadamente un tercio de la población mundial está infectada por este patógeno, siendo generalmente asintomática (Furtado, Smith, Belfort Jr, Gattey, \& Winthrop, 2011), la alta prevalencia de la infección en la población es dependiente de la edad, estudios han demostrado que la seroprevalencia de $T$. gondii en diversas poblaciones va desde un 22,5\% a más del 80\% en la población (Commodaro et al., 2009; Glasner et al., 1992).

La afectación ocular es una de las afectaciones poco comunes por $T$. gondii y se ha estimado que se produce en una proporción del 1-2\% de las personas infectadas y se asocia con un riesgo de recurrencia de por vida y perdida de la visión (London, Hovakimyan, Cubillan, Siverio Jr, \& Cunningham Jr, 2010), la Coriorretinitis toxoplásmica se ve a menudo en los entornos de la enfermedad congénita o adquirida después del nacimiento como resultado de una infección aguda (J. G. Montoya \& Remington, 1996), (Nussenblatt \& Belfort, 1994). Siendo la forma de presentación más común de la toxoplasmosis ocular la afectación típica del polo posterior de un solo ojo y las lesiones puede ser solitarias, múltiple o satélites a una cicatriz retiniana pigmentada, siendo que el $75 \%$ de los pacientes tienen cicatrices previas a en la primera consulta(Arantes et al., 2015).

La afectación ocular toma el nombre de corrioretinitis como resultado de una retinitis previa combinada con una reacción inflamatoria que compromete al epitelio pigmentario de la coroides (Dodds, 2003). La aparición de lesiones oculares varían y dependen de la duración y la intensidad lo cual puede estar relacionados con el huésped, parásito, o factores ambientales (Holland et al., 2008). El genotipo del parásito infectante parece ser un determinante importante de la gravedad de la enfermedad en pacientes inmunocompetentes (Dodds, 2003).

La toxoplasmosis ocular es una entidad cuyo diagnóstico se basa en los hallazgos oculares, acompañado de los exámenes serológicos para T. gondii (Toledo González et al., 2010). La confirmación serológica de la evidencia de infección por $T$. gondii es una parte esencial para establecer el diagnóstico de toxoplasmosis ocular. Esto permite establecer el diagnóstico en relación con el aspecto típico de las 


\section{universidâd}

lesiones oculares. La presencia de anticuerpos séricos específicos contra T. gondii es coherente y compatible con el diagnostico de toxoplasmosis ocular, pero por si misma la prueba solo establece que la persona tiene la infección (Delair et al., 2011).

\section{Caso clínico}

Paciente masculino de 19 años de edad, estudiante universitario el cual viene a la consulta con historia de que hace tres días, al despertar por la mañana noto que le amanece con el ojo derecho rojo (hiperemia conjuntival), el cual no le dolía, al día siguiente sigue igual, pero nota que la visión con el ojo derecho es un poco borrosa, al tercer día sigue igual por lo que decide ir a la clínica de la UNAN Managua de donde le dan orden para ir a consulta al Centro Nacional de Oftalmología.

El paciente en el interrogatorio refiere antecedentes familiares de Glaucoma por parte de la abuela, cataratas la abuela, pterigión el papa, no refiere haber tenido enfermedades infectocontagiosas previas, tampoco tener hábitos tóxicos (tabaco, alcohol).

Le realizan los siguientes exámenes para valorar el problema visual.

\section{Datos positivos al examen oftalmológico}

\begin{tabular}{|l|c|c|}
\hline Ojo & Agudeza visual & Tonometría \\
\hline Derecho & $20 / 26$ & 12 \\
\hline Izquierdo & $20 / 26$ & 10 \\
\hline
\end{tabular}

\section{Fondo de ojo.}

Papila de bordes bien definidos vasos arteriales y venosos de calibre normal con placa de en el sector nasal de la retina. Zona activa de retinitis.

\section{Exámenes complementarios}

Toxotest positivo títulos $1 / 256$ (positivo $>1 / 16$ )

$>$ Toxo IgM Negativo

Toxo IgG $322.6 \mathrm{UI} / \mathrm{ml}$ normal (hasta $5 \mathrm{UI} / \mathrm{ml}$ )

$>$ PCR Negativo

$>$ RPR No reactor

$>$ VSG 4 mm/hr (hasta 19) 
$>$ HIV No reactivo

$>$ HIV No reactivo

$>$ CMV IgM Negativo

> CMV IgG 7.8 UI/ml (hasta 9.8)

\section{Diagnóstico.}

Uveítis posterior del ojo derecho por Toxoplasma gondii.

\section{Tratamiento:}

Pirimetamina $25 \mathrm{mg}$ BID por un mes.

Trimetoprim sulfametoxazol (800 mg) (160 mg) BID por un mes

Cita en un mes.

El paciente regresa a la consulta al mes, refiere que ha mejorado su agudeza visual, se le hace fondo de ojo de control el cual se observa lesión de corrioretinitis cicatrizada.

\section{Revisión de la literatura sobre toxoplasmosis ocular}

\section{Epidemiología}

La infección por Toxoplasma gondii generalmente se adquiere por la ingestión de alimentos o agua contaminados con ooquistes liberados provenientes de las heces de los gatos, alimentos crudos que contienen quistes, leche no pasteurizada y huevos que contienen taquizoitos. También puede ser transmitida a través de la saliva (lamer o gotitas en el aire), esperma, transfusión de sangre, trasplante de órganos, y la placenta (transmisión congénita). (J. Montoya \& Liesenfeld, 2004), (Bonametti, Passos, Da Silva, \& Bortoliero, 1995), (Garcia et al., 1999).

La transmisión vertical de la toxoplasmosis se produce como resultado de la infección primaria (infección más raramente secundaria) en las mujeres embarazadas, cuando la enfermedad materna no se diagnostica. La infección fetal se produce en hasta 65\% -70\% y los resultados en la morbilidad infantil significativa, con lesiones oculares como la manifestación más frecuente. (Dunn et al., 1999). Aproximadamente un tercio de la población mundial está infectada por este patógeno, siendo generalmente asintomática (Furtado et al., 2011), la alta prevalencia en la población es dependiente de la 


\section{universidâd}

edad, estudios han demostrado que la seroprevalencia de $T$. gondii en diversas poblaciones va desde un 22,5\% a más del 80\% en la población (Commodaro et al., 2009; Glasner et al., 1992).

La carne destinada al consumo humano puede contener quistes y por lo tanto actuar como fuente de infección de T.gondii. Las encuestas han demostrado que más del 25\% de las ovejas y los cerdos están infectados, y el cerdo se considera la fuente más común de infección en los EE.UU. y en el sur de Brasil. Los quistes son aislados con menos frecuencia de la carne de vaca, aunque de acuerdo con una seropositividad estudio en el ganado es del 2\% -10\% (Bonametti et al., 1995)

La mayoría de los estudios que relacionan una endémica infección por T. gondii debido a agua contaminada son de países en desarrollo (Holland, 2003).

La virulencia de toxoplasmosis depende de la interacción de varios factores, incluyendo la función del sistema inmunológico, el inóculo, la etapa de infectar del parásito, y el genotipo de la $T$. gondii aislar. La cepa de tipo II es responsable de más del $70 \%$ de los casos sintomáticos en los seres humanos en Francia y en EEUU (Howe, Honoré, Derouin, \& Sibley, 1997). A pesar de la ausencia de estudios que evalúan la diferencia en la expresión de las cepas en pacientes con toxoplasmosis sistémica, estudios en animales silvestres y animales de granja han demostrado los tipos I y III a ser más frecuente que el tipo II (Cavalcante et al., 2006; Dubey et al., 2007). La cepa de tipo I parece ser responsable de la mayoría de las infecciones en oculares (Vallochi et al., 2005).

Diversos estudios epidemiológico realizados sobre los factores de riesgo para toxoplasmosis ocular recién adquirida, demuestran que la ingestión de carne cruda, poco cocida, carne seca o ahumada, frecuentes (> una vez por semana), la actividad en el jardín / patio, y el género masculino están relacionados (Jones et al., 2009; Toledo González et al., 2010; Bosch-Driessen, Berendschot, Ongkosuwito, \& Rothova, 2002)

\section{Manifestaciones oculares}

La toxoplasmosis ocular puede ser congénita o adquirida (postnatal) como resultado de la infección aguda recurrencia (Montoya \& Remington, 1996). Normalmente afecta el polo posterior de un solo ojo y las lesiones puede ser única, múltiple o satélites adyacentes a las cicatrices pigmentadas de la retina (Commodaro et al., 2009).

Las lesiones activas se presentan en forma de manchas grisáceas-blanco, de necrosis retiniana adyacente a coroiditis, además de vasculitis, hemorragia y vitritis. La curación se produce desde la 
periferia hacia el centro de la lesión, con cambios pigmentarios variables en la retina. La uveítis anterior es un hallazgo poco frecuente (Jones et al., 2006).

La retina es el sitio primario de la infección ocular por T.gondii, pero la coroides, vítreo y cámara anterior también pueden estar implicados. La coroides es afectado secundariamente; por lo tanto, las lesiones coroideas no se producen en ausencia de afectación de la retina (Holland, 2004).

La pérdida de la agudeza visual (AV) se puede producir dependiendo de la localización de la lesión y el grado de la inflamación asociada. Inicialmente, la AV puede verse comprometida por la inflamación vítrea, incluso cuando la lesión no afecta a la mácula. Las lesiones grandes y lesiones en la fóvea o región perifoveal a menudo conducen a un daño visual permanente. Sin embargo, si la región central de la fóvea no se ha lesionado, una vez que se controla la infestación ocular, la reacción inflamatoria asociada generalmente se resuelve la AV y la visión vuelve a la normalidad (Jasper et al., 2013).

En pacientes ancianos o inmunodeprimidos, la toxoplasmosis ocular puede ser bilateral y multifocal y tiende a ser más agresiva. La incidencia de afectación ocular durante la infección temprana por T. gondii suele ser mayor entre los ancianos. Pueden haber formas de presentación atípicas las cuales incluyen toxoplasmosis punteada externa de la retina, vasculitis retiniana, oclusiones vasculares retinianas, desprendimiento de retina, retinopatía pigmentaria unilateral simulando la retinitis pigmentosa, neurorretinitis, y otras formas de neuropatía óptica, necrosis de la retina periférica, y escleritis (Rey et al., 2013).

Las complicaciones oculares que se pueden presentar incluyen neo vascularización coroidea, catarata, glaucoma, atrofia del nervio óptico, y desprendimiento de retina, especialmente en los niños (Bosch-Driessen, Karimi, Stilma, \& Rothova, 2000).

Las lesiones en la toxoplasmosis ocular pueden variar en apariencia. La duración e intensidad dependen del huésped, parásito, y los factores ambientales. El genotipo del parásito infectante parece ser un factor determinante de la gravedad en pacientes inmunocompetentes (Jasper et al., 2013).

La Vasculitis retiniana y las reacciones inflamatorias asociadas pueden ser los únicos signos oftálmicos durante las primeras etapas de la toxoplasmosis ocular adquirida. El desarrollo posterior de la retinitis o cicatrices en el mismo ojo consistente con toxoplasmosis ocular sugiere parásitos como la causa de la inflamación inicial (Ganesh, Sharma, Narayana, \& Biswas, 2004). 


\section{universidâd}

La toxoplasmosis ocular recurrente no suele estar asociada con síntomas sistémicos y el riesgo de recurrencia parece ser dependiente de la edad. Sin embargo, actualmente no existe un consenso en cuanto a la edad que el riesgo es mayor. Además, se ha observado un mayor riesgo relativo en personas mayores de 40 años de edad (Holland et al., 2008), mientras que una encuesta en Suiza detectado tasas más altas en los pacientes menores de 30 años (Garweg, 2005). En Europa, la tasa de recurrencia media es de hasta un $80 \%$ en los pacientes con más de 5 años de seguimiento. Estudios realizados en los Países Bajos sugieren un mayor riesgo de recurrencia durante el primer año después de un episodio activo de toxoplasmosis ocular (Garweg, 2005). Las lesiones oculares a menudo se desarrollan años después de la infección toxoplásmica y tienden a ser asintomáticas (Nussenblatt \& Belfort, 1994). Las causas de la recurrencia de Toxoplasmosis ocular siguen sin estar claros, pero pueden estar relacionados con la ruptura de los quistes latentes ubicados en la retina (Arantes et al., 2015), o debido a la presencia de Toxoplasma gondii en la sangre periférica (Furtado et al., 2011).

La toxoplasmosis ocular aumenta el riesgo de lesión del disco óptico y se asocia con complicaciones graves tales como lesión macular, desprendimiento de retina y neo vascularización coroidea (Silveira et al., 2002).

\section{Diagnóstico}

El diagnóstico de toxoplasmosis ocular es generalmente clínico. La presencia de anticuerpos IgM indica una infección adquirida recientemente, y la IgG indica infección de vieja data, sin embargo, aún títulos altos de estos anticuerpos no pueden confirmar el diagnóstico de infección ocular. Títulos de anticuerpos IgG pueden persistir altos años después de una infección aguda, siendo la prevalencia de estos anticuerpos alta en la población general (Ongkosuwito, Bosch-Driessen, Kijlstra, \& Rothova, 2000).

La toxoplasmosis ocular se puede diagnosticar patológicamente por medio de la histología (identificación de quistes en biopsias teñidas con hematoxilina y eosina), mediante técnicas de inmunohistoquímica (detección de anticuerpos monoclonales o policlonales) (Rao \& Font, 1977), por reacción en cadena de polimerasa PCR (Brézin et al., 1990). En la histología, la toxoplasmosis se caracteriza generalmente por un extenso infiltrado inflamatorio granulomatoso de las áreas coroideas y necróticas (Belfort et al., 2009). 
El ADN de T. gondii ha sido identificado por PCR en fragmentos de tejido ocular de pacientes con presunta toxoplasmosis ocular, incluso cuando quistes tisulares típicos no fueron identificados en la histopatología (Ongkosuwito et al., 2000).

El análisis con PCR del humor vítreo de pacientes sometidos a diagnóstico diferencial de Toxoplasmosis ocular es útil, incluso cuando la presentación es atípica(Montoya, Parmley, Liesenfeld, Jaffe, \& Remington, 1999)

\section{Diagnóstico diferencial}

Retinitis por Citomegalovirus

Retinitis por CMV se produce casi exclusivamente en individuos inmunodeprimidos, particularmente aquellos con SIDA que tienen bajos CD4 ${ }^{+}$recuentos de células (<100 / mm 3). CMV produce múltiples lesiones puntiforme granulares las cuales ocasionalmente podrían asemejarse a las lesiones producidas por la toxoplasmosis (Sahu, Namperumalsamy, Walimbe, \& Rajalakshmi, 1999).

En cuanto a otras retinitis de origen viral, la PCR puede ser utilizada para definir el diagnóstico (Harper, Miller, Schiffman, \& Davis, 2009).

\section{Retinitis por sífilis}

La sífilis ha reaparecido en especial en asociación con el SIDA (Garweg, 2005), y la inflamación intraocular se estima que ocurre en el 2,5 y el 5\% de los pacientes con sífilis (Karp, Schlaeffer, Jotkowitz, \& Riesenberg, 2009), con manifestaciones del segmento posterior de la retina produciendo, coriorretinitis, lo cual pueden ser confundidos con aquellos asociados con la toxoplasmosis, independientemente de la su tamaño, por lo tanto se deben enviar pruebas treponemicas para hacer el diagnóstico diferencial (Garweg, 2005).

\section{Síndrome de múltiples puntos blancos evanescentes}

Patología que se caracteriza por presentar múltiples lesiones puntiformes retinianas profundas, mal definidas que pueden simular una coriorretinitis toxoplásmica (Harper et al., 2009).

\section{Tratamiento}

Terapia de toxoplasmosis ocular puede incluir medicamentos antimicrobianos con o sin corticosteroides sistémicos. Algunos oftalmólogos tratan todos los casos de toxoplasmosis ocular 


\section{universidâd}

independientemente de presentación, mientras que otros tratan a los pacientes sólo toxoplasmosis ocular con intensa vitritis, lesiones en el polo posterior o cerca del disco óptico, o inmunosupresión (Rothova et al, 2015).

Al igual que con otras enfermedades parasitarias, agentes farmacológicos tienen que cruzar varias barreras biológicas importantes para ser clínicamente efectivo contra $T$. gondii. Esto incluye la membrana plasmática, la membrana parasitaria, y las membranas de orgánulos específicos (más probables en el fluido intracelular).Terapias farmacológicas actuales se basan en una variedad de mecanismos (tales como la inhibición del transporte de electrones y el metabolismo de nucleótidos y la traducción) que han sido recientemente revisados en detalle.(Barna, Debache, Vock, Küster, \& Hemphill, 2013).

La pirimetamina + sulfadiazina + corticosteroides es la combinación más utilizada de medicamentos para el tratamiento de toxoplasmosis ocular y se puede considerar la terapia clásica (Montoya \& Liesenfeld, 2004).

Los pacientes con toxoplasmosis ocular activa pueden ser tratados durante 4-6 semanas con SXT, con o sin clindamicina adyuvante y prednisona. SXT parece ser un sustituto seguro y eficaz para el esquema clásico (Opremcak, Scales, \& Sharpe, 1992).

La combinación de pirimetamina y azitromicina fue tan eficaz como la combinación de pirimetamina y sulfadiazina en un ensayo prospectivo, aleatorizado. Por lo tanto, ambos son alternativas aceptables en el tratamiento de la visión que amenazan la toxoplasmosis ocular (Bosch-Driessen et al., 2002)

La evidencia reciente sugiere una amplia variación en la práctica clínica sobre el tratamiento de toxoplasmosis ocular. En una encuesta transversal con expertos uveítis, 13 (17\%) de un total de 76 encuestados reportaron el uso de corticosteroides orales en el tratamiento de la toxoplasmosis ocular en pacientes inmunocompetentes, independientemente de los hallazgos clínicos (Holland \& Lewis, 2002). Los otros informaron el uso de esteroides por sólo indicaciones específicas, tales como vitritis importante (71\%), pérdida de la visión (59\%), la proximidad de las lesiones a la fóvea o disco óptico (35\%), y las lesiones grandes (5\%). Prednisona era el corticosteroide más frecuente (97\%), que se utiliza en dosis y esquemas variados (comenzó simultáneamente con antibióticos o con un retraso de 1-7 días). El esquema más popular, aprobada por el $29 \%$ de los encuestados, fue la combinación de sulfadiazina, pirimetamina y esteroides (Holland \& Lewis, 2002). Una preferencia similar se observó en un estudio transversal más reciente con 1.000 oftalmólogos (Lum, Jones, Holland, \& Liesegang, 2005). Sin embargo, en 2 ensayos 
clínicos aleatorizados Se encontró una diferencia estadísticamente significativa en el resultado visual funcional entre los grupos tratados con sulfadiazina + pirimetamina esteroides y grupos + tratados con SXT o pirimetamina + azitromicina (Bosch-Driessen et al., 2002).

La controversia sobre el uso de los corticosteroides en toxoplasmosis ocular no es nueva. Mientras que muchos oftalmólogos los utilizan, a partir de hasta 3-7 días después del inicio de la terapia antiparasitaria, otros evitan dar por completo. El daño ocular se ha atribuido a la inflamación intraocular resultante de lesión del tejido causada por el parásito. En vista de sus propiedades anti-inflamatorias, los corticosteroides son probablemente beneficiosos para los pacientes con toxoplasmosis ocular activa. Los estudios en animales han demostrado esteroides para ser un complemento útil, minimizando el daño ocular. Por ejemplo, no se observó enfermedad sistémica o recurrencia de la inflamación ocular en conejos infectados crónicamente tratados con hidrocortisona (Bosch-Driessen \& Rothova, 1998).

En los pacientes con recurrencia frecuente, el tratamiento a largo plazo con 1 comprimido de sulfametoxazol $(800 \mathrm{mg})$ y trimetoprim $(160 \mathrm{mg}) 3$ veces a la semana reduce la tasa de recurrencia del $23,8 \%$ al 6,6\%. Un reciente estudio prospectivo, aleatorizado, ensayos clínicos y doble ciego mostró que la terapia SXT intermitente (1 comprimido cada dos días) redujo la recurrencia toxoplasmosis ocular en un $100 \%$ en 1 año después del tratamiento, pero no produjo ningún efecto medible en mejor corregida agudeza visual, posiblemente debido a la insuficiente tamaño de la muestra. Los episodios repetidos de toxoplasmosis ocular pueden causar pérdida de la visión a largo plazo (Lima, Saraiva, \& Saraiva, 2015).

La combinación de clindamicina y atovacuona (o azitromicina) puede proporcionar un método seguro para el tratamiento agudo de toxoplasmosis ocular en pacientes embarazadas, siempre y cuando la duración del tratamiento se ajuste de acuerdo a la respuesta. Definen "respuesta positiva al tratamiento" como una disminución en el tamaño de las lesiones retinocoroidales y disminución de la opacidad vitrea. El tratamiento debe ser extendido en pacientes inmunocompetentes por lo menos 30-60 días. Los pacientes deben comenzar con corticosteroides orales dentro de las 48 horas después de la iniciación de la terapia antimicrobiana de intensa. Las sulfonamidas deben evitarse en el tercer trimestre, ya que compiten con la bilirrubina de proteínas séricas, causando kernicterus.

La pirimetamina es potencialmente teratogénico y debe evitarse, especialmente en el primer trimestre. Un enfoque clínico multidisciplinario puede ser necesaria en el tratamiento de mujeres embarazadas con toxoplasmosis sistémica. Se recomienda la colaboración con un especialista de Enfermedades Infecciosas y Obstetricas (Lima et al., 2015). 


\section{universidâd}

En los pacientes con contraindicaciones para la terapia sistémica, pueden ser tratados con inyecciones intravítreas de clindamicina y esteroides. (Sobrin, Kump, \& Foster, 2007) Demostraron una asociación entre la inyección clindamicina y la resolución de toxoplasmosis ocular. Del mismo modo, con las inyecciones intravítreas de clindamicina y dexametasona.

Las inyecciones subconjuntivales de clindamicina parecen ser una interesante alternativa al esquema clásico.Por otra parte, (Martinez, Zhang, Conway, \& Peyman, 1998). Martínez et al. Obtuvieron muy buenos resultados visuales en una serie de casos de mujeres embarazadas con toxoplasmosis ocular activa tratados con una combinación de clindamicina intraocular y dexametasona (Martinez et al., 1998).

\section{CONCLUSIÓN}

La toxoplasmosis es una infección de muy alta prevalencia a nivel mundial siendo está en la mayoría de los infectado asintomática y autolimitada, una de sus formas de presentación la afectación ocular, aunque es de muy baja prevalencia es importante un diagnostico precoz, para así mismo dar tratamiento y evitar que el paciente desarrolle complicaciones que puedan llevar consigo a la perdida de la visión.

\section{REFERENCIAS}

Arantes, Tiago EF, Silveira, C., Holland, G. N, Muccioli, C., Yu Fei, J. Jeffrey L., \& Belfort, R. (2015). Ocular involvement following postnatally acquired Toxoplasma gondii infection in southern Brazil: a 28-year experience. American journal of ophthalmology, 159(6), 1002-1012. e1002. https://doi.org/10.1016/j.ajo.2015.02.015

Barna, F., Debache, K., Vock, C. A, Küster, T., \& Hemphill, A. (2013). In vitro effects of novel ruthenium complexes in Neospora caninum and Toxoplasma gondii tachyzoites. Antimicrobial agents and chemotherapy, 57(11), 5747-5754. https://doi.org/10.1128/AAC.02446-12

Belfort, R. N, Fernandes, B.F., Romano, A., Nose, R., Cools-Lartigue, J., Navajas, E. V, .. Jr, R. Belfort. (2009). Bilateral macular hemorrhage as a complication of drug-induced anemia: a case report. Journal of medical case reports, 3(1), 16. https://doi.org/10.1186/1752-1947-3-16 
Bonametti, A.M., Passos, Jdo N, Da Silva, E.M., \& Bortoliero, A.L. (1995). [Outbreak of acute toxoplasmosis transmitted thru the ingestion of ovine raw meat]. Revista da Sociedade Brasileira de Medicina Tropical, 30(1), 21-25. https://doi.org/10.1590/S0037-86821997000100005

Bosch-Driessen, E.H., \& Rothova, A. (1998). Sense and nonsense of corticosteroid administration in the treatment of ocular toxoplasmosis. British journal of ophthalmology, 82(8), 858-860. https://doi.org/10.1136/bjo.82.8.858

Bosch-Driessen, L. E.H, Berendschot, Tos T.J.M., Ongkosuwito, J. V, \& Rothova, A. (2002). Ocular toxoplasmosis: clinical features and prognosis of 154 patients. Ophthalmology, 109(5), 869-878. https://doi.org/10.1016/S0161-6420(02)00990-9

Bosch-Driessen, L. H., Karimi, S., Stilma, J.S., \& Rothova, A. (2000). Retinal detachment in ocular toxoplasmosis. Ophthalmology, 107(1), 36-40. https://doi.org/10.1016/S0161-6420(99)00013-5

Bosch-Driessen, L. H., Verbraak, F. D., Suttorp-Schulten, M.S.A., Van Ruyven, R.L.J, Klok, Anne Marie, Hoyng, Carel B, \& Rothova, Aniki. (2002). A prospective, randomized trial of pyrimethamine and azithromycin vs pyrimethamine and sulfadiazine for the treatment of ocular toxoplasmosis. American journal of ophthalmology, 134(1), 34-40. https://doi.org/10.1016/S00029394(02)01537-4

Brézin, A.P., Egwuagu, C. E, Burnier, M., Silveira, C., Mahdi, R. M., Gazzinelli, R.T., ... Nussenblatt, R. B. (1990). Identification of Toxoplasma gondii in paraffin-embedded sections by the polymerase chain reaction. American journal of ophthalmology, 110(6), 599-604. https://doi.org/10.1016/S0002-9394(14)77055-2

Cavalcante, GT, Aguiar, DM, Camargo, L M A, Labruna, MB, De Andrade, HF, Meireles, LR, . . . Gennari, SM. (2006). Seroprevalence of Toxoplasma gondii antibodies in humans from rural Western Amazon, Brazil. Journal of Parasitology, 92(3), 647-649. https://doi.org/10.1645/GE$\underline{774 \mathrm{R} .1}$

Commodaro, Alessandra G, Belfort, Rubens N, Rizzo, Luiz Vicente, Muccioli, Cristina, Silveira, Claudio, Burnier Jr, Miguel N, \& Belfort Jr, Rubens. (2009). Ocular toxoplasmosis: an update and review of the literature. Memórias do Instituto Oswaldo Cruz, 104(2), 345-350. https://doi.org/10.1590/S0074-02762009000200030

Delair, Emmanuelle, Latkany, Paul, Noble, A Gwendolyn, Rabiah, Peter, McLeod, Rima, \& Brézin, Antoine. (2011). Clinical manifestations of ocular toxoplasmosis. Ocular immunology and inflammation, 19(2), 91-102. https://doi.org/10.3109/09273948.2011.564068 


\section{universidâd}

Dodds, Emilio M. (2003). Toxoplasmosis ocular. Archivos de la Sociedad Española de Oftalmología, 78(10), 531-541. https://doi.org/10.4321/S0365-66912003001000004

Dubey, JP, Gennari, SM, Sundar, N, Vianna, M C B, Bandini, LM, Yai, L E O, . . Su, C. (2007). Diverse and atypical genotypes identified in Toxoplasma gondii from dogs in São Paulo, Brazil. Journal of Parasitology, 93(1), 60-64. https://doi.org/10.1645/GE-972R.1

Dunn, David, Wallon, Martine, Peyron, François, Petersen, Eskild, Peckham, Catherine, \& Gilbert, Ruth. (1999). Mother-to-child transmission of toxoplasmosis: risk estimates for clinical counselling. The Lancet, 353(9167), 1829-1833. https://doi.org/10.1016/S0140-6736(98)08220-8

Furtado, João M, Smith, Justine R, Belfort Jr, Rubens, Gattey, Devin, \& Winthrop, Kevin L. (2011). Toxoplasmosis: a global threat. Journal of global infectious diseases, 3(3), 281. https://doi.org/10.4103/0974-777X.83536

Ganesh, Sudha K, Sharma, Sumita, Narayana, Kannan M, \& Biswas, Jyotirmay. (2004). Fuchs\&CloseCurlyQuote; heterochromic iridocyclitis following bilateral ocular toxoplasmosis. Ocular immunology and inflammation, 12(1), 75-77. https://doi.org/10.1076/ocii.12.1.75.28065

Garweg, JG. (2005). Determinants of immunodiagnostic success in human ocular toxoplasmosis. Parasite immunology, 27(3), 61-68. https://doi.org/10.1111/j.1365-3024.2005.00747.x

Garweg, JG. (2005). Determinants of immunodiagnostic success in human ocular toxoplasmosis. Parasite immunology, 27(3), 61-68. https://doi.org/10.1111/j.1365-3024.2005.00747.x

Glasner, Peter D, Silveira, Claudio, Kruszon-Moran, Deanna, Martins, Maria C, Burnier, Miguel, Silveira, Silvia, . . Belfort, Rubens. (1992). An unusually high prevalence of ocular toxoplasmosis in southern Brazil. American journal of ophthalmology, 114(2), 136-144. https://doi.org/10.1016/S0002-9394(14)73976-5

Harper, Thomas W, Miller, Darlene, Schiffman, Joyce C, \& Davis, Janet L. (2009). Polymerase chain reaction analysis of aqueous and vitreous specimens in the diagnosis of posterior segment infectious uveitis. American journal of ophthalmology, 147(1), 140-147. https://doi.org/10.1016/i.ajo.2008.07.043

Holland, Gary N. (2003). Ocular toxoplasmosis: a global reassessment. American Journal of Ophthalmology, 136(6), 973-988. https://doi.org/10.1016/j.ajo.2003.09.040

Holland, Gary N. (2004). Ocular toxoplasmosis: a global reassessment: part II: disease manifestations and management. American journal of ophthalmology, 137(1), 1-17. https://doi.org/10.1016/S0002$\underline{9394(03) 01319-9}$ 
Holland, Gary N, Crespi, Catherine M, ten Dam-van Loon, Ninette, Charonis, Alexander C, Yu, Fei, Bosch-Driessen, Lotje H, \& Rothova, Aniki. (2008). Analysis of recurrence patterns associated with toxoplasmic retinochoroiditis. American journal of ophthalmology, 145(6), 1007-1013. e1001. https://doi.org/10.1016/i.ajo.2008.01.023

Holland, Gary N, \& Lewis, Kevan G. (2002). An update on current practices in the management of ocular toxoplasmosis 1, 2. American journal of ophthalmology, 134(1), 102-114. https://doi.org/10.1016/S0002-9394(02)01526-X

Howe, Daniel K, Honoré, Stephanie, Derouin, Francis, \& Sibley, L David. (1997). Determination of genotypes of Toxoplasma gondii strains isolated from patients with toxoplasmosis. Journal of clinical microbiology, 35(6), 1411-1414.

Jasper, Smitha, Vedula, Satyanarayana S, John, Sheeja S, Horo, Saban, Sepah, Yasir J, \& Nguyen, Quan Dong. (2013). Corticosteroids as adjuvant therapy for ocular toxoplasmosis. The Cochrane Library. https://doi.org/10.1002/14651858.CD007417.pub2

Jones, J. L., Dargelas, V., Roberts, J., Press, C., Remington, J. S., \& Montoya, J. G. (2009). Risk factors for Toxoplasma gondii infection in the United States. Clinical Infectious Diseases, 49(6), 878-884. https://doi.org/10.1086/605433

Glasner, Peter D, Silveira, Claudio, Kruszon-Moran, Deanna, Martins, Maria C, Burnier, Miguel, Silveira, Silvia, . . Belfort, Rubens. (1992). An unusually high prevalence of ocular toxoplasmosis in southern Brazil. American journal of ophthalmology, 114(2), 136-144. https://doi.org/10.1016/S0002-9394(14)73976-5

Harper, Thomas W, Miller, Darlene, Schiffman, Joyce C, \& Davis, Janet L. (2009). Polymerase chain reaction analysis of aqueous and vitreous specimens in the diagnosis of posterior segment infectious uveitis. American Journal of Ophthalmology, 147(1), 140-147. e142. https://doi.org/10.1016/j.ajo.2008.07.043

Holland, Gary N. (2003). Ocular toxoplasmosis: a global reassessment. American Journal of Ophthalmology, 136(6), 973-988.https://doi.org/10.1016/j.ajo.2003.09.040

Holland, Gary N. (2004). Ocular toxoplasmosis: a global reassessment: part II: disease manifestations and management. American journal of ophthalmology, 137(1), 1-17. https://doi.org/10.1016/S0002-9394(03)01319-9

Holland, Gary N, Crespi, Catherine M, ten Dam-van Loon, Ninette, Charonis, Alexander C, Yu, Fei, Bosch-Driessen, Lotje H, \& Rothova, Aniki. (2008). Analysis of recurrence patterns associated 


\section{universidâd}

with toxoplasmic retinochoroiditis. American journal of ophthalmology, 145(6), 1007-1013. e1001. https://doi.org/10.1016/j.ajo.2008.01.023

Holland, Gary N, \& Lewis, Kevan G. (2002). An update on current practices in the management of ocular toxoplasmosis 1, 2. American journal of ophthalmology, 134(1), 102-114. https://doi.org/10.1016/S0002-9394(02)01526-X

Howe, Daniel K, Honoré, Stephanie, Derouin, Francis, \& Sibley, L David. (1997). Determination of genotypes of Toxoplasma gondii strains isolated from patients with toxoplasmosis. Journal of clinical microbiology, 35(6), 1411-1414.

Jasper, Smitha, Vedula, Satyanarayana S, John, Sheeja S, Horo, Saban, Sepah, Yasir J, \& Nguyen, Quan Dong. (2013). Corticosteroids as adjuvant therapy for ocular toxoplasmosis. The Cochrane Library. https://doi.org/10.1002/14651858.CD007417.pub2

Jones, J. L., Dargelas, V., Roberts, J., Press, C., Remington, J. S., \& Montoya, J. G. (2009). Risk factors for Toxoplasma gondii infection in the United States. Clinical Infectious Diseases, 49(6), 878-884. https://doi.org/10.1086/605433

Jones, J. L., Muccioli, C., Belfort Jr, R., Holland, G. N , Roberts, J. M, \& Silveira, C. (2006). Recently acquired Toxoplasma gondii infection, Brazil. Emerging infectious diseases, 12(4), 582. https://doi.org/10.3201/eid1204.051081

Lima Guilherme, S. C., Saraiva, P. G. C., \& Saraiva, F. P. (2015). Current Therapy of Acquired Ocular Toxoplasmosis: A Review. Journal of Ocular Pharmacology and Therapeutics.

London, N.J., Hovakimyan, A., Cubillan, L. D., Siverio Jr, C. D, \& Cunningham Jr, E. T. (2010). Prevalence, clinical characteristics, and causes of vision loss in patients with ocular toxoplasmosis. European journal of ophthalmology, 21(6), 811-819. https://doi.org/10.5301/EJO.2011.6403

Lum, F., Jones, J. L, Holland, G. N, \& Liesegang, T. J. (2005). Survey of ophthalmologists about ocular toxoplasmosis. American journal of ophthalmology, 140(4), 724. e721-724. e729. https://doi.org/10.1016/j.ajo.2005.04.048

Martinez, C. E, Zhang, D., Conway, M. D., \& Peyman, G. A. (1998). Successful management of ocular toxoplasmosis during pregnancy using combined intraocular clindamycin and dexamethasone with $\begin{array}{lllll}\text { systemic sulfadiazine. } & \text { International }\end{array}$ https://doi.org/10.1023/A:1006129422690

Montoya, J.G. \& Liesenfeld, O. (2004). Toxoplasmosis Lancet 363, 1965-1976. CrossRef, PubMed, CAS, Web of Science ${ }^{\circledR}$ Times Cited, 807. 
Montoya, J. G, Parmley, S., Liesenfeld, O., Jaffe, G. J. \& Remington, J. S. (1999). Use of the polymerase chain reaction for diagnosis of ocular toxoplasmosis. Ophthalmology, 106(8), 1554-1563. https://doi.org/10.1016/S0161-6420(99)90453-0

Montoya, J. G, \& Remington, J. S. (1996). Toxoplasmic chorioretinitis in the setting of acute acquired $\begin{array}{llll}\text { toxoplasmosis. } \quad \text { Clinical infectious } & \text { 237-282. }\end{array}$ https://doi.org/10.1093/clinids/23.2.277

Nussenblatt, R. B, \& Belfort, R. (1994). Ocular toxoplasmosis: an old disease revisited. Jama, 271(4), 304-307. https://doi.org/10.1001/jama.1994.03510280066035 https://doi.org/10.1001/jama.271.4.304

Ongkosuwito, J.V, Bosch-Driessen, E.H, Kijlstra, A., \& Rothova, A.A. (2000). Serologic evaluation of patients with primary and recurrent ocular toxoplasmosis for evidence of recent infection. Evidence-Based Ophthalmology, 1(2), 90-91. https://doi.org/10.1097/00132578-200001000$\underline{00014}$

Opremcak, E M., Scales, D. K., \& Sharpe, M. R. (1992). Trimethoprim-sulfamethoxazole therapy for ocular toxoplasmosis. Ophthalmology, 99(6), 920-925. https://doi.org/10.1016/S0161$\underline{6420(92) 31873-1}$

Rao, N. A, \& Font, R.L. (1977).Toxoplasmic retinochoroiditis:electron-microscopic and immunofluorescence studies of formalin-fixed tissue. Archives of ophthalmology, 95(2), 273-277. https://doi.org/10.1001/archopht.1977.04450020074012

Rey, A., Llorenç, V., Pelegrín, L., Mesquida, M., Molins, B., Rios, J., . . \& Adán, A. (2013). Clinical pattern of toxoplasmic retinochoroiditis in a spanish referral center. Ophthalmologica, 229(3), 173178. https://doi.org/10.1159/000348740

Rothova, A, Buitenhuis, H.J, Meenken, C., Baarsma, G.S, Boen-Tan, T.N, de Jong, P.T.V.M., . . Zaal, M.J.W. (2015). Therapy of ocular toxoplasmosis. International ophthalmology, 13(6), 415-419. https://doi.org/10.1007/BF02306491

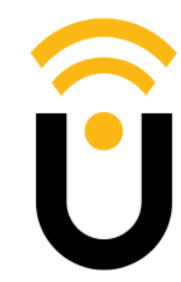

